\title{
Robotic partial nephrectomy versus radical nephrectomy in elderly patients with large renal masses
}

\author{
Alessandro VECCIA 1, 2, Paolo DELL'OGLIO 3, Alessandro ANTONELLI 4, \\ Andrea MINERVINI 5, Giuseppe SIMONE ${ }^{6}$, Benjamin CHALLACOMBE ${ }^{7}$, Sisto PERDONÀ 8, \\ James PORTER ${ }^{9}$, Chao ZHANG ${ }^{10}$, Umberto CAPITANIO ${ }^{11}$, Chandru P. SUNDARAM ${ }^{12}$, \\ Giovanni CACCIAMANI 13, Monish ARON 13, Uzoma ANELE 1, Lance J. HAMPTON 1, \\ Claudio SIMEONE 2, Geert DE NAEYER ${ }^{3}$, Aaron BRADSHAWH ${ }^{14}$, Andrea MARI 5 , \\ Riccardo CAMPI 5, Marco CARINI 5, Cristian FIORI 15, Michele GALLUCCI ${ }^{6}$, \\ Ken JACOBSOHN ${ }^{16}$, Daniel EUN ${ }^{17}$, Clayton LAU ${ }^{18}$, Jihad KAOUK ${ }^{19}$, Ithaar DERWEESH ${ }^{14}$, \\ Francesco PORPIGLIA ${ }^{15}$, Alexandre MOTTRIE ${ }^{3}$, Riccardo AUTORINO 1 *
}

${ }^{1}$ Division of Urology, Virginia Commonwealth University Health System, Richmond, VA, USA; 2 Unit of Urology, Department of Medical and Surgical Specialties, Radiological Science, and Public Health, ASST Spedali Civili Hospital, University of Brescia, Brescia, Italy; ${ }^{3}$ ORSI Academy, Melle, and Department of Urology, OLV Ziekenhuis, Aalst, Belgium; ${ }^{4}$ Department of Urology, University of Verona, Verona, Italy; ${ }^{5}$ Department of Urology, Careggi Hospital, University of Florence, Florence, Italy; 'Department of Urology, "Regina Elena" National Cancer Institute, Rome, Italy; ${ }^{7} \mathrm{MRC}$ Centre for Transplantation, NIHR Biomedical Research Centre, Guy's Hospital, King's College, London, UK; ${ }^{8}$ Unit of Urology, IRCCS Fondazione "G. Pascale", Naples, Italy; ${ }^{5}$ Swedish Urology Group, Swedish Medical Center, Seattle, WA, USA; ${ }^{0}$ Department of Urology, Changhai Hospital, Shanghai Shi, China; ${ }^{11}$ Unit of Urology, Division of Experimental Oncology, Urological Research Institute (URI), San Raffaele Hospital IRCCS, Milan, Italy; ${ }^{2}$ Department of Urology, Indiana University Health, Indianapolis, IN, USA; ${ }^{13}$ USC Institute of Urology, Catherine and Joseph Aresty Department of Urology, Keck School of Medicine, University of Southern California, Los Angeles, CA, USA; ${ }^{14}$ Department of Urology, UC San Diego Health System, La Jolla, CA, USA; ${ }^{15}$ Department of Urology, San Luigi Gonzaga Hospital, University of Turin, Turin, Italy; ${ }^{6}$ Department of Urology, Medical College Wisconsin, Milwaukee, WI, USA; ${ }^{17}$ Department of Urology, Lewis Katz School of Medicine at Temple University, Philadelphia, PA, USA; ${ }^{18}$ Division of Urology, City of Hope National Medical Center, Duarte, CA, USA; ${ }^{19}$ Department of Urology, Glickman Urological \& Kidney Institute, Cleveland Clinic, Cleveland, OH, USA

*Corresponding author: Riccardo Autorino, Division of Urology, VCU Medical Center, PO Box 980118, Richmond, VA 23298-0118, USA. E-mail: ricautor@gmail.com

\section{A B S T R A C T}

BACKGROUND: Recent evidence suggests that the "oldest old" patients might benefit of partial nephrectomy (PN), but decision-making for this subset of patients is still controversial. Aim of this study is to compare outcomes of robotic partial (RPN) or radical nephrectomy (RRN) for large renal masses in patients older than 65 years.

METHODS: We identified 417 $\geq 65$ years old patients who underwent RRN or RPN for cT1b or $\geq \mathrm{cT} 2$ renal mass at 17 high volume centers. Propensity score match analysis was performed adjusting for age, ASA $\geq 3$, pre-operative eGFR, and clinical tumor size. Predictors of complications, functional and oncological outcomes were evaluated in multivariable logistic and Cox regression models.

RESULTS: After propensity score analysis, 73 patients in the RPN group were matched with 74 in the RRN group. R.E.N.A.L. Score $(9.6 \pm 1.7$ vs. $8.6 \pm 1.7 ; \mathrm{P}<0.001)$, and high complexity $(56$ vs. $15 \% ; \mathrm{P}=0.001$ ) were higher in the RRN Estimated blood loss was higher in the RPN group $(200$ vs. $100 \mathrm{~mL} ; \mathrm{P}<0.001)$. RPN showed higher rate of overall complications ( 38 vs. $23 \%$; $\mathrm{P}=0.05)$, but not major complications $(\mathrm{P}=0.678)$. At last follow-up, RPN group showed better 


\section{COPYRIGHT $^{\odot} 2020$ EDIZIONI MINERVA MEDICA}

functional outcomes both in eGFR $(55.4 \pm 22.6$ vs. $45.7 \pm 15.7 \mathrm{~mL} / \mathrm{min} ; \mathrm{P}=0.016)$ and lower eGFR variation $(9.7$ vs. 23.0 $\mathrm{mL} / \mathrm{min} ; \mathrm{P}<0.001$ ). The procedure type was not associated with recurrence free survival (RFS) (HR: $0.47 ; \mathrm{P}=0.152)$ and overall mortality (OM) (0.22; $\mathrm{P}=0.084)$.

CONCLUSIONS: RPN in elderly patients with large renal masses provides acceptable surgical, and oncological outcomes allowing better functional preservation relative to RRN. The decision to undergo RPN in this subset of patients should be tailored on a case by case basis.

(Cite this article as: Veccia A, Dell'Oglio P, Antonelli A, Minervini A, Simone G, Challacombe B, et al. Robotic partial nephrectomy versus radical nephrectomy in elderly patients with large renal masses. Minerva Urol Nefrol 2020;72:99-108. DOI: $10.23736 / \mathrm{S} 0393-2249.19 .03583-5)$

KEY WORDS: Nephrectomy; Aged; Patient outcome assessment.

$C_{t i a}$ urrent urological guidelines recommend partial nephrectomy $(\mathrm{PN})$ as the elective treatment for cT1a renal masses, whereas radical nephrectomy $(\mathrm{RN})$ remains the primary option for cT1b and $\geq$ cT2 tumors. ${ }^{1,2}$ However, PN for large renal masses has begun to be considered a valid and safe treatment option in terms of oncological control, and improved functional outcomes also for larger renal masses. ${ }^{3}$ The widespread adoption of robotic surgery and its well-known technical advantages in terms of visualization and handling of the anatomical structures favored the use of robot-assisted PN (RPN) for large renal masses as well.4, 5 In addition, cardiovascular, ${ }^{6}$ and oncological outcomes 7 indicate that PN could also be suitable for elderly patients, who are usually more likely to be elected to RN. ${ }^{8}, 9$ Furthermore, recent evidence suggests that the "oldest old" patients might benefit of PN in terms of surgical functional outcomes, and oncological safety. ${ }^{10-13}$ However, the rate of elderly patients who underwent PN for large renal mass (cT1b or $\geq \mathrm{cT} 2$ ) in these studies was underrepresented. Moreover, available evidence on robotic radical nephrectomy (RRN) in elderly patients is poor and scarce and without adequate comparison to RPN.

Therefore, the aim of this study was to assess the feasibility and safety of RPN in a large cohort of patients aged $\geq 65$ with large renal masses and to compare the perioperative, functional, and intermediate-term oncological outcomes of RPN $v s$. RRN in this subset of patients, for whom decision-making is still controversial.

\section{Materials and methods}

Institutional review board approval and data sharing was obtained at each center involved. All data for the present study were retrieved from a multi-institutional multinational database. Overall, 417 patients 65 years or older who underwent RRN or RPN for $\mathrm{cT} 1 \mathrm{~b}$ or $\geq \mathrm{cT} 2$ renal mass were included.

Baseline patient characteristics (age at the surgery, gender, body mass index [BMI], ASA Score $\geq 3$, diabetes, hypertension, chronic kidney disease $\geq$ class III, preoperative $\mathrm{Hb}$, and estimated glomerular filtration rate [eGFR], and solitary kindey status), clinical staging (tumor size, R.E.N.A.L. Score [continuous and categorical], $\mathrm{cT}, \mathrm{cN}+, \mathrm{cM}+$ ), surgical outcomes (transperitoneal approach, operative time [OT], estimated blood loss [EBL], intraoperative transfusions, complications [intraoperative, overall, $\leq 30$ days, major according to Clavien-Dindo classification $\geq 3$ ], length of stay [LoS], re-admission rate within 30 days, and $\mathrm{Hb}$ at discharge), pathological outcomes (tumor size, benign histology, pT, $\mathrm{pN}+$, Fuhrman grade $\geq 3$, positive surgical margins [PSM]), functional outcomes (eGFR and $\triangle \mathrm{eGFR}$ at discharge, 6,12 , and last follow-up) recurrence free survival (RFS) and overall survival (OS) were assessed.

\section{Statistical analysis}

In order to account for any potential baseline differences among the two groups, adjustment was performed using a 1:1 nearest-neighbor propensity score-matching. ${ }^{14}$

Propensity scores were computed using a logistic regression model to account for all measurable potential confounders. The quality of the matching was assessed through the pstest and psgraph commands. An acceptable matching quality was achieved: before matching $B=94.6 \%$, after matching $\mathrm{B}=22 \%$ (Figure 1 ). 


\section{COPYRIGHT $^{\odot} 2020$ EDIZIONI MINERVA MEDICA}

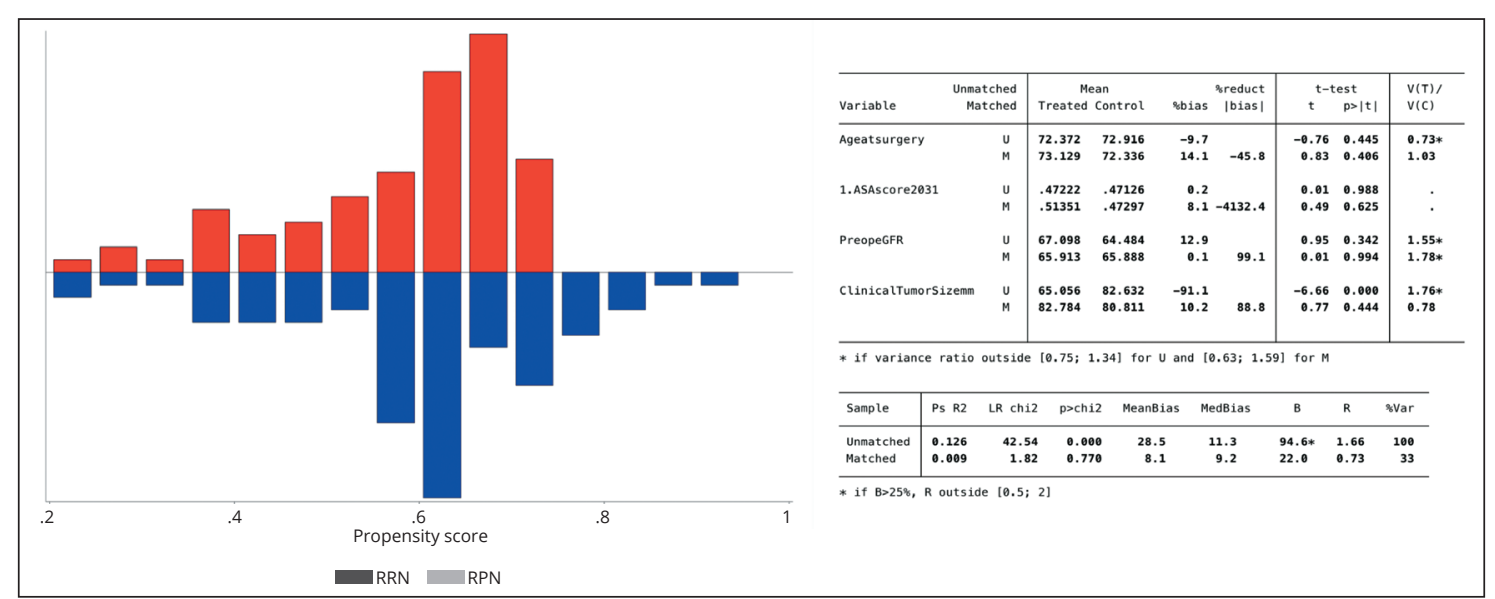

Figure 1.-Propensity score matching quality.

Statistical analysis was conducted according to guidelines ${ }^{15}$ and consisted in three steps. First, Shapiro-Wilk Test was used to establish the distribution of the data. Median (interquartile range [IQR]) was used to report not-parametric data, while mean \pm standard deviation (SD) was adopted for parametric data. Proportions were used to report categorical data. To compare the differences in the distribution of continuous and categorical variables between cases treated with RPN and RRN, Mann-Whitney U-test and Fisher's exact were used, respectively. When continuous variables showed parametric distribution, Student's $t$-test was used.

Three separate sets of multivariable logistic and Cox regression models were fitted to evaluate the effect of the type of procedure (RPN $v s$. RRN) on overall complications, eGFR decrease $\geq 25 \%$ (at discharge, 6 and 12 months, and at last follow-up), and RFS and OS. In multivariable models testing for overall complication, adjustment variables consisted of age, ASA $\geq 3$, R.E.N.A.L. Score, type of procedure (RPN vs. RRN), intraoperative transfusion, and intraoperative complications. In multivariable models testing for eGFR decrease $\geq 25 \%$, adjustment variables were age, $\mathrm{ASA} \geq 3$, preoperative eGFR, R.E.N.A.L. Score, and type of procedure (RPN vs. RRN). In multivariable models testing for recurrence and survival, adjustment variables consisted of R.E.N.A.L. Score and type of procedure (RPN vs. RRN). All statistical tests were performed with Stata ${ }^{\circledR} 15.0$ (StataCorp 2017.
Stata Statistical Software: release 15. StataCorp LLC, College Station, TX, USA), and statistical significance was set at $\mathrm{P} \leq 0.05$.

\section{Results}

Baseline characteristics of study cohort before matching

Before matching 143 and 273 patients were found in the RRN and RPN, respectively. The baseline $\mathrm{Hb}$ level was higher in the RPN group: 13.9 (12.5-15.1) vs. 12.8 (11.7-14.0) g/dL; $\mathrm{P}<0.001$. The RPN group demonstrated smaller tumor size (7.0 vs. $8.1 \mathrm{~cm} ; \mathrm{P}<0.001)$, and lower R.E.N.A.L. Score $(8.0$ vs. $10.0 ; \mathrm{P}<0.001)$ and fewer high complexity masses (30 vs. 58\%; $\mathrm{P}<0.001)$. Patients undergoing $\mathrm{RRN}$ had a higher rate of clinically advanced disease ( $\mathrm{cT} \geq 3: 13 \mathrm{vs}$. $9 \% ; \mathrm{P}<0.001-\mathrm{cN}+8$ vs. $1 \% ; \mathrm{P}=0.004-\mathrm{cM}+19$ vs. $3 \% ; \mathrm{P}<0.001)$.

Baseline characteristics of study cohort after matching

After propensity score analysis 73 patients in the RPN group were matched with 74 in the RRN one. No statistically significant difference was found in terms of baseline features and tumor dimensions between RPN and RRN groups $(\mathrm{P}=0.685)$. However, the R.E.N.A.L. Score (9.6 \pm 1.7 vs. $8.6 \pm 1.7 ; \mathrm{P}<0.001)$, and proportion of high-complexity tumors (56 vs. $15 \%$; $\mathrm{P}=0.001$ ) were higher in the RRN group compared to RPN. 


\section{COPYRIGHT $^{\odot} 2020$ EDIZIONI MINERVA MEDICA}

VECCIA

PARTIAL $V S$. RADICAL NEPHRECTOMY IN PATIENTS WITH LARGE RENAL MASSES

TABLE I.-Patients'baseline features and outcomes.

\begin{tabular}{|c|c|c|c|c|c|c|}
\hline \multirow[b]{2}{*}{ Variables ${ }^{f}$} & \multicolumn{3}{|c|}{ Before matching } & \multicolumn{3}{|c|}{ After matching } \\
\hline & RRN & RPN & $P$ value & RRN & RPN & $P$ value \\
\hline Number of patients & 143 & 273 & & 74 & 73 & \\
\hline \multicolumn{7}{|l|}{ Baseline features } \\
\hline Age (years) & $72.0(68.0-77.0)$ & $72.0(68.0-76.0)$ & 0.308 & $71.3(67.7-77.0)$ & $73.0(68.0-77.0)$ & 0.388 \\
\hline Gender (male) & 95/143 (66\%) & 195/273 (71\%) & 0.313 & $50 / 74(67 \%)$ & $50 / 73(68 \%)$ & 1.000 \\
\hline BMI $\left(\mathrm{kg} / \mathrm{m}^{2}\right)$ & $27.7(24.5-30.2)$ & $26.9(24.6-30.0)$ & 0.350 & $27.8(24.8-31.0)$ & $26.3(24.3-30.3)$ & 0.284 \\
\hline $\mathrm{ASA} \geq 3$ & $63 / 114(55 \%)$ & $107 / 226(47 \%)$ & 0.135 & $35 / 74(47 \%)$ & $38 / 73(52 \%)$ & 0.622 \\
\hline Diabetes & $38 / 142(27 \%)$ & $63 / 242(26 \%)$ & 0.905 & $22 / 73(30 \%)$ & $24 / 69(35 \%)$ & 0.594 \\
\hline Hypertension & $85 / 142(60 \%)$ & $126 / 242(52 \%)$ & 0.167 & $43 / 73(59 \%)$ & $34 / 69(49 \%)$ & 0.312 \\
\hline $\mathrm{CKD} \geq \mathrm{III}$ & 16/141 (11\%) & $41 / 223(18 \%)$ & 0.077 & $11 / 72(15 \%)$ & $16 / 69(23 \%)$ & 0.286 \\
\hline $\mathrm{Hb}$ at baseline $(\mathrm{g} / \mathrm{dL})$ & $12.8(11.7-14.0)$ & $13.9(12.5-15.1)$ & $<0.001$ & $13.0(12.1-14.5)$ & $13.6(12.5-14.6)$ & 0.111 \\
\hline $\begin{array}{l}\text { eGFR at baseline }(\mathrm{mL} / \\
\left.\mathrm{min} / 1.73 \mathrm{~m}^{2}\right)^{\neq}\end{array}$ & $64.5 \pm 20.2$ & $67.4 \pm 22.0$ & 0.216 & $65.8 \pm 17.4$ & $66.1 \pm 23.3$ & 0.939 \\
\hline Solitary kidney & $1 / 121(1 \%)$ & $12 / 235(5 \%)$ & 0.069 & $1 / 74(1 \%)$ & $5 / 73(7 \%)$ & 0.116 \\
\hline \multicolumn{7}{|l|}{ Clinical tumor staging } \\
\hline Tumor size $(\mathrm{cm})$ & $8.1(7.2-9.8)$ & $7.0(5.0-8.0)$ & $<0.001$ & $8.0(7.1-9.0)$ & $8.0(7.3-8.6)$ & 0.685 \\
\hline $\begin{array}{l}\text { R.E.N.A.L. } \\
\text { (continuous) }\end{array}$ & $10.0(9.0-11.0)$ & $8.0(7.0-10.0)$ & $<0.001$ & $9.6 \pm 1.7 \neq$ & $8.6 \pm 1.7 \neq$ & $<0.001$ \\
\hline R.E.N.A.L. (complexity) & & & $<0.001$ & & & 0.001 \\
\hline Low (4-6) & $4 / 122(3 \%)$ & $59 / 245(24 \%)$ & & $3 / 68(4 \%)$ & $10 / 73(14 \%)$ & \\
\hline Intermediate (7-9) & $47 / 122(39 \%)$ & $113 / 245(46 \%)$ & & $27 / 68(40 \%)$ & $37 / 73(51 \%)$ & \\
\hline $\operatorname{High}(10-12)$ & $71 / 122(58 \%)$ & $73 / 245(30 \%)$ & & $38 / 68(56 \%)$ & $11 / 73(15 \%)$ & \\
\hline $\mathrm{cT}$ & & & $<0.001$ & & & 0.222 \\
\hline $1 \mathrm{~b}$ & $15 / 135(11 \%)$ & $143 / 272(53 \%)$ & & 6/66 (9\%) & $10 / 73(14 \%)$ & \\
\hline $2 \mathrm{a}$ & $74 / 135(55 \%)$ & $88 / 272(32 \%)$ & & $41 / 66(62 \%)$ & $46 / 73(63 \%)$ & \\
\hline $2 b$ & $26 / 135(19 \%)$ & $16 / 272(6 \%)$ & & $5 / 66(8 \%)$ & 9/73 (12\%) & \\
\hline $3 a$ & $17 / 135(13 \%)$ & 25/272 (9\%) & & $12 / 66(18 \%)$ & $8 / 73(11 \%)$ & \\
\hline $3 b$ & $2 / 135(1 \%)$ & - & & $2 / 66(3 \%)$ & - & \\
\hline 4 & $1 / 135(1 \%)$ & - & & - & - & \\
\hline $\mathrm{cN}+$ & $12 / 143(8 \%)$ & $5 / 248(1 \%)$ & 0.004 & $5 / 74(9 \%)$ & $2 / 71(3 \%)$ & 0.442 \\
\hline $\mathrm{cM}+$ & $27 / 143(19 \%)$ & $7 / 264(3 \%)$ & $<0.001$ & $10 / 74(13 \%)$ & $3 / 73(4 \%)$ & 0.078 \\
\hline \multicolumn{7}{|l|}{ Surgical outcomes } \\
\hline Transperitoneal & $119 / 123(97 \%)$ & $215 / 262(82 \%)$ & $<0.001$ & $69 / 70(98 \%)$ & $54 / 72(75 \%)$ & $<0.001$ \\
\hline $\mathrm{OT}(\mathrm{min})$ & $174.0(136.0-225.0)$ & $160.0(120.0-224.0)$ & 0.049 & $160.0(120.0-210.0)$ & $158.0(115.0-240.0)$ & 0.872 \\
\hline $\mathrm{EBL}(\mathrm{mL})$ & $100.0(50.0-200.0)$ & $150.0(100.0-300.0)$ & $<0.001$ & $100(50.0-150.0)$ & $200.0(100.0-475.0)$ & $<0.001$ \\
\hline $\begin{array}{l}\text { Intraoperative } \\
\text { transfusions }\end{array}$ & $6 / 143(4 \%)$ & $15 / 255(6 \%)$ & 0.614 & $1 / 74(1 \%)$ & $5 / 72(7 \%)$ & 0.114 \\
\hline $\begin{array}{l}\text { Intraoperative } \\
\text { complications }\end{array}$ & $10 / 100(10 \%)$ & $18 / 242(7 \%)$ & 0.277 & $3 / 67(4 \%)$ & $8 / 72(11 \%)$ & 0.211 \\
\hline Overall complications & $26 / 125(20 \%)$ & $58 / 243(24 \%)$ & 0.600 & $17 / 74(23 \%)$ & $28 / 73(38 \%)$ & 0.050 \\
\hline Major complications ${ }^{\dagger}$ & 4/19(21\%) & 9/47 (19\%) & 1.000 & $3 / 13(23 \%)$ & $4 / 24(16 \%)$ & 0.678 \\
\hline Length of stay (days) & $3.0(2.0-4.0)$ & $4.0(3.0-6.0)$ & $<0.001$ & $3.0(2.0-5.5)$ & $5.0(4.0-7.0)$ & $<0.001$ \\
\hline Re-admission 30 days & $7 / 107(6 \%)$ & $6 / 178(3 \%)$ & 0.227 & $4 / 66(6 \%)$ & $3 / 58(5 \%)$ & 1.000 \\
\hline $\mathrm{Hb}$ at discharge $(\mathrm{g} / \mathrm{dL})$ & $11.4(10.1-12.4)$ & $10.9(10.0-12.5)$ & 0.470 & $11.5 \pm 1.5^{t}$ & $10.5 \pm 1.4$ & $<0.001$ \\
\hline \multicolumn{7}{|l|}{ Pathological outcomes } \\
\hline Tumor size $(\mathrm{cm})$ & $8.0(6.5-9.9)$ & $6.0(4.8-7.8)$ & $<0.001$ & $7.6 \pm 2.3$ & $7.2 \pm 1.9$ & 0.231 \\
\hline Benign tumor & $5 / 143(3 \%)$ & $50 / 299(17 \%)$ & 0.044 & $5 / 74(7 \%)$ & $11 / 73(15 \%)$ & 0.120 \\
\hline $\mathrm{pT} \geq 3$ & $72 / 143(50 \%)$ & $72 / 273(26 \%)$ & $<0.001$ & $40 / 74(54 \%)$ & $23 / 73(31 \%)$ & 0.008 \\
\hline $\mathrm{pN}+$ & $8 / 140(6 \%)$ & - & $<0.001$ & $4 / 72(5 \%)$ & - & $<0.001$ \\
\hline Fuhrman grade $\geq 3$ & $70 / 124(56 \%)$ & 73/191 (38\%) & 0.001 & $32 / 64(50 \%)$ & $30 / 55(54 \%)$ & 0.046 \\
\hline PSM & $11 / 142(7 \%)$ & $18 / 281(6 \%)$ & 0.539 & $10 / 70(14 \%)$ & $7 / 73(9 \%)$ & 0.445 \\
\hline \multicolumn{7}{|l|}{ Functional outcomes } \\
\hline $\begin{array}{l}\text { eGFR at discharge } \\
\left(\mathrm{mL} / \mathrm{min} / 1.73 \mathrm{~m}^{2}\right)\end{array}$ & $46.0(37.0-56.9)$ & $60.9(41.6-71.7)$ & $<0.001$ & $46.0(37.5-58.6)$ & $57.8(38.9-70.3)$ & 0.023 \\
\hline $\begin{array}{l}\Delta \mathrm{eGFR} \text { at discharge } \\
\left(\mathrm{mL} / \mathrm{min} / 1.73 \mathrm{~m}^{2}\right)\end{array}$ & $12.5(5.6-28.0)$ & $10.1(1.29-20.0)$ & 0.027 & $15.2(6.0-29.3)$ & $10.9(1.0-23.0)$ & 0.084 \\
\hline $\begin{array}{l}\text { eGFR at } 6 \text { months }(\mathrm{mL} / \\
\left.\mathrm{min} / 1.73 \mathrm{~m}^{2}\right)\end{array}$ & $46.0(39.0-52.5)$ & $52.1(41.0-65.7)$ & 0.050 & $46.0(39.0-55.7)$ & $51.9(41.1-66.4)$ & 0.201 \\
\hline $\begin{array}{l}\Delta \mathrm{eGFR} \text { at } 6 \text { months } \\
\left(\mathrm{mL} / \mathrm{min} / 1.73 \mathrm{~m}^{2}\right)\end{array}$ & $19.0(10.0-28.0)$ & $10.9(3.9-20.7)$ & 0.006 & $19.0(10.0-29.0)$ & $11.5(3.3-19.8)$ & 0.032 \\
\hline $\begin{array}{l}\text { eGFR at } 12 \text { months } \\
\left(\mathrm{mL} / \mathrm{min} / 1.73 \mathrm{~m}^{2}\right)\end{array}$ & $45.4(42.0-58.7)$ & $57.8(39.9-70.6)$ & 0.023 & $46.2(42.0-59.0)$ & $51.9(34.0-66.4)$ & 0.713 \\
\hline
\end{tabular}

(To be continued) 


\section{COPYRIGHT $^{\circledR} 2020$ EDIZIONI MINERVA MEDICA}

PARTIAL VS. RADICAL NEPHRECTOMY IN PATIENTS WITH LARGE RENAL MASSES

VECCIA

TABLE I.-Patients' baseline features and outcomes (continues).

\begin{tabular}{|c|c|c|c|c|c|c|}
\hline \multirow[b]{2}{*}{ Variables $^{f}$} & \multicolumn{3}{|c|}{ Before matching } & \multicolumn{3}{|c|}{ After matching } \\
\hline & RRN & RPN & $P$ value & RRN & RPN & $P$ value \\
\hline $\begin{array}{l}\triangle \mathrm{eGFR} \text { at } 12 \text { months } \\
\left(\mathrm{mL} / \mathrm{min} / 1.73 \mathrm{~m}^{2}\right)\end{array}$ & $16.0(6.7-27.0)$ & $8.0(1.2-10.1)$ & 0.203 & $16.0(8.0-27.0)$ & $12.4(2.6-20.5)$ & 0.262 \\
\hline $\begin{array}{l}\text { eGFR at last follow-up } \\
\left(\mathrm{mL} / \mathrm{min} / 1.73 \mathrm{~m}^{2}\right)\end{array}$ & $46.0(37.0-55.0)$ & $61.0(42.6-75.0)$ & $<0.001$ & $45.7 \pm 15.7 \neq$ & $55.4 \pm 22.6^{t}$ & 0.016 \\
\hline $\begin{array}{l}\Delta \mathrm{eGFR} \text { at last follow-up } \\
\left(\mathrm{mL} / \mathrm{min} / 1.73 \mathrm{~m}^{2}\right)\end{array}$ & $22.0(11.0-29.7)$ & $8.0(1.2-18.1)$ & $<0.001$ & $23.0(11.0-31.0)$ & $9.7(0.7-19.9)$ & $<0.001$ \\
\hline $\begin{array}{l}\text { Follow-up length } \\
\text { (months) }\end{array}$ & $14.5(6.6-33.8)$ & $22.0(6.0-42.0)$ & 0.139 & $12.0(8.0-27.0)$ & $30.0(12.0-43.0)$ & $<0.001$ \\
\hline $\begin{array}{l}\mathrm{eGFR} \leq 45 \mathrm{~mL} / \\
\mathrm{min} / 1.73 \mathrm{~m}^{2} \\
\text { at } 6 \text { months }\end{array}$ & $30 / 61(49 \%)$ & $30 / 86(35 \%)$ & 0.059 & $25 / 51(49 \%)$ & $14 / 37(38 \%)$ & 0.385 \\
\hline $\begin{array}{l}\text { eGFR } \leq 45 \mathrm{~mL} / \\
\mathrm{min} / 1.73 \mathrm{~m}^{2} \\
\text { at last follow-up }\end{array}$ & $41 / 83(49 \%)$ & 48/167 (29\%) & 0.001 & $24 / 46(50 \%)$ & $20 / 55(36 \%)$ & 0.225 \\
\hline
\end{tabular}

\section{Surgical outcomes}

Regarding surgical outcomes, the transperitoneal approach was preferred with greater prevalence among the RRN group (98 vs. 75\%; $\mathrm{P}<0.001)$. EBL was higher in the RPN group (200.0 [100.0-475.0] mL vs. 100 [50.0-150.0] $\mathrm{mL} ; \mathrm{P}<0.001)$, but no difference regarding intraoperative transfusions were observed $(\mathrm{P}=0.114)$. Patients undergoing RPN had a higher rate of overall complications ( 38 vs. $23 \% ; \mathrm{P}=0.05$ ), but there was no difference between groups for major complications $(\mathrm{P}=0.678)$. RPN patients had longer $\operatorname{LoS}(3.0$ vs. 5.0 days; $\mathrm{P}<0.001)$ (Table I).

In multivariable logistic regression analysis, the type of the procedure did not reach the independent predictor status (OR: 2.22; 95\% CI: $0.95,5.19 ; \mathrm{P}=0.064$; Table II).

\section{Histopathological and functional outcomes}

The RRN group was found to have more advanced disease as evidenced by a higher rate of $\mathrm{pT} \geq 3$ (54\% vs. $31 \% ; \mathrm{P}=0.008)$. The $\mathrm{RPN}$ group demonstrated a higher eGFR at discharge [57.8 (38.9-70.3) vs. 46.0 (37.5-58.6) $\mathrm{mL} / \mathrm{min}$; $\mathrm{P}=0.023]$. At 6 months, eGFR variation was lower in the RPN group [11.5 (3.3-19.8) vs. 19.0 (10.0-29.0) $\mathrm{mL} / \mathrm{min} ; \mathrm{P}=0.032]$. At last followup, the RPN group maintained a higher eGFR (55.4 \pm 22.6 vs. $45.7 \pm 15.7 \mathrm{~mL} / \mathrm{min} ; \mathrm{P}=0.016)$ and lower eGFR variation (9.7 [0.7-19.9] vs. 23.0 [11.0-31.0] $\mathrm{mL} / \mathrm{min} ; \mathrm{P}<0.001)$. Median followup was 19.5 (IQR: 8-40.5) months (Table I, Figure 2). On multivariable logistic regression analysis, RPN reached the independent predictor status for eGFR decrease $\geq 25 \%$ at discharge (OR:

TABLE II._Logistic regression of predictors of overall complications.

\begin{tabular}{|c|c|c|c|c|c|c|c|c|c|c|c|c|}
\hline \multirow[b]{3}{*}{ Variables } & \multicolumn{6}{|c|}{ Before matching } & \multicolumn{6}{|c|}{ After matching } \\
\hline & \multicolumn{3}{|c|}{ Univariate analysis } & \multicolumn{3}{|c|}{ Multivariate analysis } & \multicolumn{3}{|c|}{ Univariate analysis } & \multicolumn{3}{|c|}{ Multivariate analysis } \\
\hline & OR & $95 \% \mathrm{CI}$ & $P$ value & OR & $95 \% \mathrm{CI}$ & $\mathrm{P}$ value & OR & $95 \% \mathrm{CI}$ & $P$ value & OR & $95 \% \mathrm{CI}$ & $\mathrm{P}$ value \\
\hline Age & 0.96 & $0.92,1.00$ & 0.107 & 0.95 & $0.89,1.00$ & 0.086 & 0.97 & $0.91,1.03$ & 0.343 & 0.95 & $0.88,1.01$ & 0.155 \\
\hline $\mathrm{ASA} \geq 3$ & 2.43 & $1.41,4.18$ & 0.001 & 3.44 & $1.83,6.46$ & $<0.001$ & 2.74 & $1.31,5.71$ & 0.007 & 3.65 & $1.55,8.59$ & 0.003 \\
\hline R.E.N.A.L. Score & 1.07 & $0.94,1.22$ & 0.263 & 1.07 & $0.91,1.26$ & 0.352 & 1.00 & $0.81,1.23$ & 0.979 & 1.00 & $0.95,5.19$ & 0.978 \\
\hline \multicolumn{13}{|l|}{ Type of procedure } \\
\hline RRN & Ref & & & Ref & & & Ref & & & Ref & & \\
\hline RPN & 1.19 & $0.70,2.01$ & 0.507 & 1.43 & $0.70,2.91$ & 0.316 & 2.08 & $1.01,4.27$ & 0.045 & 2.22 & $0.95,5.19$ & 0.064 \\
\hline $\begin{array}{l}\text { Intraoperative } \\
\text { transfusions }\end{array}$ & 1.31 & $0.45,3.80$ & 0.615 & 0.64 & $0.14,2.83$ & 0.560 & 1.16 & $0.20,6.61$ & 0.862 & 0.56 & $0.78,4.02$ & 0.566 \\
\hline $\begin{array}{l}\text { Intraoperative } \\
\text { complications }\end{array}$ & 1.62 & $0.63,4.15$ & 0.310 & 0.87 & $0.23,3.25$ & 0.897 & 1.40 & $0.38,5.08$ & 0.604 & 0.97 & $0.23,4.06$ & 0.975 \\
\hline
\end{tabular}




\section{COPYRIGHT $^{\circledR} 2020$ EDIZIONI MINERVA MEDICA}

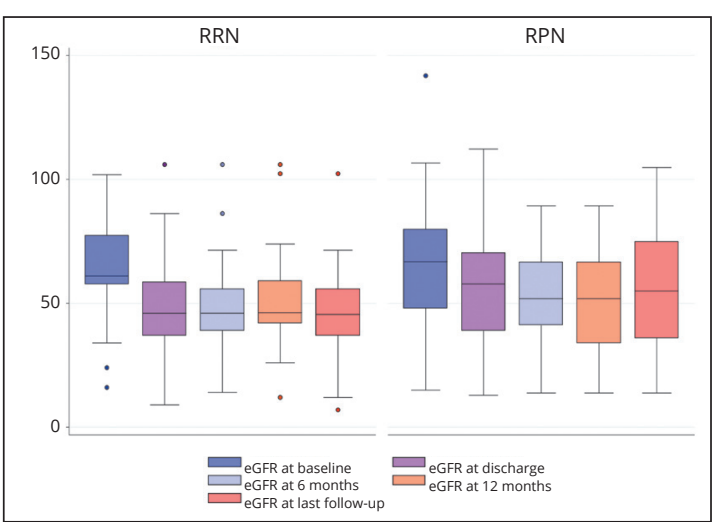

Figure 2.- - eGFR values time distribution.

$0.33 ; 95 \%$ CI: $0.15,0.73 ; \mathrm{P}=0.007)$, at 6 months (OR: $0.23 ; 95 \%$ CI: $0.08,0.67 ; \mathrm{P}=0.007$ ), and at last follow-up (OR: 0.29; 95\% CI: 0.12, 0.72; $\mathrm{P}=0.008$; Table III).

\section{Oncologic outcomes}

Using multivariable Cox regression analysis, the type of the procedure was not associated with RFS (HR: 0.47; 95\% CI: 0.17, 1.31; $\mathrm{P}=0.152$; Table IV) and OM (0.22; 95\% CI: 0.04, 1.21; $\mathrm{P}=0.084$; Table IV). R.E.N.A.L. Score was found to be associated with shorter RFS (HR: 1.40; 95\% CI: 1.02, 1.91; $\mathrm{P}=0.033$; Table IV).

\section{Discussion}

To the best of our knowledge this study represents the largest report available to date on elderly patients treated with RPN for large renal masses, and the first one providing a comparison between RPN and RRP in this subset of patients. Our analysis revealed some interesting findings which may contribute to the debate on

TABLE III.-Logistic regression of predictors of eGFR decrease $\geq 25 \%$.

\begin{tabular}{|c|c|c|c|c|c|c|c|c|c|c|c|c|}
\hline \multirow[b]{3}{*}{ Variables } & \multicolumn{6}{|c|}{ Before matching } & \multicolumn{6}{|c|}{ After matching } \\
\hline & \multicolumn{3}{|c|}{ Univariate analysis } & \multicolumn{3}{|c|}{ Multivariate analysis } & \multicolumn{3}{|c|}{ Univariate analysis } & \multicolumn{3}{|c|}{ Multivariate analysis } \\
\hline & OR & $95 \% \mathrm{CI}$ & $P$ value & OR & $95 \% \mathrm{CI}$ & $P$ value & OR & $95 \% \mathrm{CI}$ & $\mathrm{P}$ value & OR & $95 \% \mathrm{CI}$ & $\mathrm{P}$ value \\
\hline \multicolumn{13}{|c|}{ eGFR decrease $\geq 25 \%$ at discharge } \\
\hline Age & 1.02 & $0.95,1.04$ & 0.931 & 1.02 & $0.96,1.08$ & 0.475 & 1.03 & $0.97,1.09$ & 0.328 & 1.03 & $0.96,1.04$ & 0.391 \\
\hline $\mathrm{ASA} \geq 3$ & 0.90 & $0.54,1.53$ & 0.720 & 1.05 & $0.56,2.00$ & 0.858 & 0.81 & $0.41,1.62$ & 0.568 & 1.22 & $0.54,2.75$ & 0.625 \\
\hline Preoperative eGFR & 1.01 & $1.00,1.03$ & 0.003 & 1.02 & $1.00,1.04$ & 0.002 & 1.02 & $1.00,1.04$ & 0.004 & 1.03 & $1.01,1.05$ & 0.004 \\
\hline R.E.N.A.L. Score & 1.10 & $0.96,1.25$ & 0.159 & 1.03 & $0.88,1.21$ & 0.677 & 1.03 & $0.83,1.23$ & 0.895 & 0.99 & $0.79,1.25$ & 0.998 \\
\hline \multicolumn{13}{|l|}{ Type of procedure } \\
\hline $\mathrm{RN}$ & Ref & & & & & & Ref & & & & & \\
\hline $\mathrm{PN}$ & 0.42 & $0.25,0.70$ & 0.001 & 0.26 & $0.14,0.52$ & $<0.001$ & 0.42 & $0.20,0.84$ & $<0.001$ & 0.33 & $0.15,0.73$ & 0.007 \\
\hline \multicolumn{13}{|c|}{ eGFR decrease $\geq 25 \%$ at 6 months } \\
\hline Age & 1.00 & $0.94,1.06$ & 0.849 & 0.99 & $0.92,1.06$ & 0.856 & 1.00 & $0.93,1.08$ & 0.819 & 0.99 & $0.91,1.07$ & 0.833 \\
\hline $\mathrm{ASA} \geq 3$ & 1.02 & $0.52,2.02$ & 0.933 & 1.33 & $0.58,3.04$ & 0.489 & 0.97 & $0.41,2.26$ & 0.953 & 1.06 & $0.38,2.97$ & 0.901 \\
\hline Pre-operative eGFR & 1.02 & $1.00,1.04$ & 0.007 & 1.03 & $1.00,1.05$ & 0.006 & 1.01 & $0.99,1.04$ & 0.094 & 1.02 & $0.99,1.04$ & 0.110 \\
\hline R.E.N.A.L. Score & 0.99 & $0.83,1.19$ & 0.966 & 0.86 & $0.69,1.07$ & 0.203 & 0.98 & $0.76,1.25$ & 0.879 & 0.87 & $0.64,1.17$ & 0.367 \\
\hline \multicolumn{13}{|l|}{ Type of procedure } \\
\hline $\mathrm{RN}$ & Ref & & & Ref & & & Ref & & & Ref & & \\
\hline $\mathrm{PN}$ & 0.29 & $0.15,0.59$ & 0.001 & 0.21 & $0.09,0.50$ & $<0.001$ & 0.27 & $0.11,0.67$ & 0.005 & 0.23 & $0.08,0.67$ & 0.007 \\
\hline \multicolumn{13}{|c|}{ eGFR decrease $\geq 25 \%$ at 12 months } \\
\hline Age & 1.10 & $1.03,1.19$ & 0.005 & 1.11 & $1.02,1.20$ & 0.013 & 1.07 & $0.98,1.17$ & 0.111 & 1.06 & $0.97,1.17$ & 0.180 \\
\hline $\mathrm{ASA} \geq 3$ & 1.29 & $0.61,2.70$ & 0.494 & 1.41 & $0.57,3.46$ & 0.447 & 1.23 & $0.48,3.14$ & 0.658 & 1.42 & $0.47,4.28$ & 0.526 \\
\hline Pre-operative eGFR & 1.01 & $0.99,1.03$ & 0.052 & 1.01 & $0.99,1.04$ & 0.095 & 1.01 & $0.99,1.04$ & 0.199 & 1.01 & $0.99,1.04$ & 0.281 \\
\hline R.E.N.A.L. Score & 0.95 & $0.78,1.17$ & 0.684 & 0.90 & $0.71,1.15$ & 0.428 & 0.83 & $0.64,1.09$ & 0.201 & 0.83 & $0.61,1.14$ & 0.263 \\
\hline \multicolumn{13}{|l|}{ Type of procedure } \\
\hline $\mathrm{RN}$ & Ref & & & Ref & & & Ref & & & Ref & & \\
\hline $\mathrm{PN}$ & 0.45 & $0.21,0.95$ & 0.038 & 0.34 & $0.14,0.86$ & 0.024 & 0.77 & $0.30,1.99$ & 0.598 & 0.58 & $0.19,1.77$ & 0.344 \\
\hline \multicolumn{13}{|c|}{ eGFR decrease $\geq 25 \%$ at last follow-up } \\
\hline Age & 1.04 & $0.99,1.09$ & 0.073 & 1.04 & $0.97,1.10$ & 0.205 & 1.02 & $0.95,1.10$ & 0.449 & 1.02 & $0.95,1.10$ & 0.477 \\
\hline $\mathrm{ASA} \geq 3$ & 0.71 & $0.40,1.25$ & 0.241 & 0.70 & $0.34,1.44$ & 0.342 & 0.51 & $0.23,1.14$ & 0.105 & 0.64 & $0.24,1.69$ & 0.371 \\
\hline Pre-operative eGFR & 1.00 & $0.99,1.02$ & 0.188 & 1.00 & $0.99,1.02$ & 0.341 & 1.01 & $0.99,1.03$ & 0.240 & 1.00 & $0.98,1.03$ & 0.430 \\
\hline R.E.N.A.L. Score & 1.21 & $1.04,1.40$ & 0.013 & 1.03 & $0.85,1.24$ & 0.747 & 0.97 & $0.77,1.24$ & 0.859 & 0.91 & $0.70,1.18$ & 0.495 \\
\hline \multicolumn{13}{|l|}{ Type of procedure } \\
\hline $\mathrm{RN}$ & Ref & & & Ref & & & Ref & & & Ref & & \\
\hline $\mathrm{PN}$ & 0.16 & $0.09,0.29$ & $<0.001$ & 0.17 & $0.08,0.36$ & $<0.001$ & 0.28 & $0.12,0.64$ & 0.003 & 0.29 & $0.12,0.72$ & 0.008 \\
\hline
\end{tabular}




\section{COPYRIGHT $^{\circledR} 2020$ EDIZIONI MINERVA MEDICA}

PARTIAL VS. RADICAL NEPHRECTOMY IN PATIENTS WITH LARGE RENAL MASSES

VECCIA

TABLE IV.-Cox regression of predictors of RFS and OM.

\begin{tabular}{|c|c|c|c|c|c|c|}
\hline \multirow[b]{2}{*}{ Variables } & \multicolumn{3}{|c|}{ Recurrence free survival } & \multicolumn{3}{|c|}{ Overall survival } \\
\hline & HR & $95 \%$ CI & $P$ value & HR & $95 \% \mathrm{CI}$ & $P$ value \\
\hline R.E.N.A.L. Score & 1.40 & $1.02,1.91$ & 0.033 & 1.35 & $0.85,2.14$ & 0.194 \\
\hline \multicolumn{7}{|l|}{ Type of procedure } \\
\hline $\mathrm{RN}$ & Ref & & & Ref & & \\
\hline $\mathrm{PN}$ & 0.47 & $0.17,1.31$ & 0.152 & 0.22 & $0.04,1.21$ & 0.084 \\
\hline
\end{tabular}

RN: radical nephrectomy; PN: partial nephrectomy; HR: hazard ratio; CI: Confidence Interval.

nephron sparing surgery (NSS) for large (cT1b and cT2) tumors.

After propensity score analysis, we found no statistically significant difference in terms of baseline features, even for $\mathrm{CKD} \geq$ stage III and solitary kidney. This data mirrors previous literature regarding PN on RN performed for large renal masses. Mir et al. described similar findings within a systematic review and meta-analysis of 21 case-control studies on this topic. ${ }^{3}$ However, in our analysis we noticed a clinical trend to favor RPN over RRN in patients with $\mathrm{CKD} \geq$ stage III $(23 \% v s .15 \%)$ or solitary kidney ( $7 \%$ vs. $1 \%)$. In addition, recent evidence that NSS might provide better cancer specific mortality may warrant consideration for large renal masses even in an elective setting. 6,16

Contrary to previous reports, we found no statistically significant difference regarding clinical tumor size; however, RRN was mostly performed for complex tumors. Given the median age of our cohort, one might argue that this could be consequence of the patients age, who are usually managed with RN than PN due to concerns of surgical complications. ${ }^{8}$ Nevertheless, NSS for large renal masses is challenging in every subset of patients, and tumor complexity is one of the main factors considered during pre-operative planning. Despite this, NSS for high complexity tumors has shown to be feasible in experienced hands. Indeed, Buffi et al. evaluated the outcomes of 255 patients who underwent RPN at tertiary referral centers in a multicenter design study and achieved optimal surgical outcomes in $158(62 \%)$ patients. ${ }^{17}$ Their findings were consistent with ours, despite the lack of an elderly population. In fact, even if we found RPN to be associated with higher EBL, the difference with RRN was of only $100 \mathrm{~mL}$ which is clinically insignificant. To confirm this data there was no statistically significant difference in terms of intraoperative transfusions.

In our cohort we noticed a higher rate of overall complications in the RPN group, but no difference in major complications. This rate of complications is likely owed to the more complex nature of the RPN procedure during which the risk is increased by the tumor resection and reconstructive phase. Indeed, tumor excision might provoke pelvicalyceal system effraction, and vascular injury, especially during challenging procedures. ${ }^{18}$ Our data showed that the only 4 major complications in the RPN group were 3 urine leakage that required ureteral stent placement, and 1 abdominal bleeding which was managed with endovascular embolization. In addition, we found that ASA Score $\geq 3$, but no surgical technique, was the only factor associated with the risk of overall complications. Overall, our data suggest that RPN is feasible and potentially safe, but treatment decision between RPN and RRN must be balanced between outcomes, patients comorbidities, and quality of life which is more important than life expectancy itself in elderly patients. ${ }^{19,} 20$ In addition, the higher rate of overall complications rate translated into longer LoS in the RPN, another important factor to be considered in elderly patients. $^{21}$

Regarding pathological outcomes, patients undergoing RRN were found to have more advanced disease. This data is consistent with that of a recent report assessing outcomes of $\mathrm{RN}$ and PN within the RESURGE Project. In that study the authors assessed 1226 patients older than 75 years and found higher rate of $\mathrm{pT} \geq 3$ in those cases who underwent RN. ${ }^{12}$ Again, Venkatramani et al. assessed outcomes of RN and PN for cT1bcT2 within the National Cancer Database. After propensity score analysis the authors matched 


\section{COPYRIGHT $^{\circledR} 2020$ EDIZIONI MINERVA MEDICA}

6072 patients per each group showing higher rate of $\mathrm{pT} \geq 3$ in the $\mathrm{RN}$ group $(13.1 \%$ vs. $6.9 \%$; $\mathrm{P}<0.001) .{ }^{22}$ In our data the overall rate of $\mathrm{pT} \geq 3$ was higher for both groups (RPN 31\% vs. RRN $54 \%$ ), but this difference could be explained by the different covariates selection to build the propensity model.

When comparing RPN and RRN, the functional outcomes assessment deserves attention. RPN provided better kidney function compared to RRN, especially at medium and long-term follow-up. Indeed, the advantages of NSS surgery were not immediately obvious, whereas at last follow-up eGFR and eGFR variation were better for the RPN group. Nevertheless, the difference was of only about $10 \mathrm{~mL} / \mathrm{min}$ and may not be clinically significant, but this could be the case in this subset of patients whose kidney function could be already impaired. ${ }^{23}$ Of note, on multivariable analysis RPN showed a protective role regarding eGFR decrease $\geq 25 \%$. These data mirror literature evidence which largely assessed the functional advantages of nephrons unit preservation. A recent report compared function results of 250 living donors and 118 partial nephrectomy patients. The authors evaluated \% eGFR variation at discharge, 1-7 months, and last follow-up, and achieved the conclusion that PN provided immediate better functional outcomes and that should be considered, even at expense of longer ischemia time. ${ }^{24}$ If this could be true for small renal masses, it might be different for large and complex ones. Recently, Wu et al. conducted a refined analysis of the functional impact of the vascularized parenchymal mass preserved with partial nephrectomy and predictors. The authors identified four possible scenarios (1-4) based upon the percentage of parenchymal mass preserved after PN. The analysis demonstrated that high tumor complexity and dimension were related to suboptimal preservation of nephrons due to the larger resection and reconstruction. This could impair functional recovery despite NSS. ${ }^{25}$ Given these findings, our study demonstrated RPN to provide better functional outcomes for large and complex renal masses in the subset of elderly patients, as already underlined in other reports. ${ }^{11,12}$

Survival outcomes demonstrated RFS to be associated to R.E.N.A.L. Score, whereas surgical procedure (RPN or RRN) was not found to be a predictor. This data corroborates those of previous studies assessing R.E.N.A.L. Score as predictor of malignancy, more advanced pathology, and recurrence. Nagahara et al. demonstrated that high R.E.N.A.L. Score is associated with RFS (HR: 9.05; $\mathrm{P}=0.0019) .{ }^{26}$

\section{Limitations of the study}

To the best of our knowledge this is the largest report describing the outcomes of elderly patients after RPN or RRN for large renal masses. This analysis is strengthened by the propensity score design and the sample size. Despite this, it is not devoid of limitations. First, the retrospective nature introduces biases which cannot be avoided. Even if these were limited with the propensity score matched analysis, it presented a bias of $22 \%$. Moreover, the aim to balance the two groups according to surgical technique reduced the numerosity of the two groups. A second important limitation is the absence of others fragility scores to assess patient's performance status and life expectancy. This analysis cannot be generalized to all clinical realities because all the procedures were performed in high volume centers with high proficiency in robotic and renal surgery. Kidney function was evaluated through eGFR which could be misleading because of the compensatory function of the contralateral kidney. ${ }^{27,} 28$ Last, but not least, the follow-up period could be too short to evaluate accurately survival outcomes. Moreover, the follow-up length was significantly longer in patients undergoing RRN. As such, our findings on oncological outcomes should be interpreted with caution. Notwithstanding these limitations, this report provides further data regarding the management of large renal masses even in elderly population.

\section{Conclusions}

RPN in elderly patients with large renal masses offers acceptable surgical, functional, and oncological outcomes compared to RRN. The decision to undergo RPN in this subset of patients should be tailored on a case by case basis. 


\section{COPYRIGHT $^{\circ} 2020$ EDIZIONI MINERVA MEDICA}

PARTIAL VS. RADICAL NEPHRECTOMY IN PATIENTS WITH LARGE RENAL MASSES

\section{References}

1. Campbell S, Uzzo RG, Allaf ME, Bass EB, Cadeddu JA, Chang A, et al. Renal mass and localized renal cancer: AUA Guideline. J Urol 2017;198:520-9.

2. Ljungberg B, Bensalah K, Canfield S, Dabestani S, Hofmann F, Hora M, et al. EAU guidelines on renal cell carcinoma: 2014 update. Eur Urol 2015;67:913-24.

3. Mir MC, Derweesh I, Porpiglia F, Zargar H, Mottrie A, Autorino R. Partial nephrectomy versus radical nephrectomy for clinical T1b and T2 renal tumors: a systematic review and meta-analysis of comparative studies. Eur Urol 2017;71:606-17.

4. Bertolo R, Autorino R, Simone G, Derweesh I, Garisto JD, Minervini A, et al. Outcomes of robot-assisted partial nephrectomy for clinical T2 renal tumors: a multicenter analysis (ROSULA Collaborative Group). Eur Urol 2018;74:226-32.

5. Cacciamani GE, Medina LG, Gill T, Abreu A, Sotelo R, Artibani W, et al. Impact of surgical factors on robotic partial nephrectomy outcomes: comprehensive systematic review and meta-analysis. J Urol 2018;200:258-74.

6. Capitanio U, Larcher A, Cianfalone F, Trevisani F, Nini A, Mottrie A, et al. Hypertension and cardiovascular morbidity following surgery for kidney cancer. Eur Urol Oncol 2019. [Epub ahead of print].

7. Antonelli A, Minervini A, Sandri M, Bertini R, Bertolo R, Carini M, et al. Below safety limits, every unit of glomerular filtration rate counts: assessing the relationship between renal function and cancer-specific mortality in renal cell carcinoma. Eur Urol 2018;74:661-7.

8. Tan HJ, Daskivich TJ, Shirk JD, Filson CP, Litwin MS, Hu JC. Health status and use of partial nephrectomy in older adults with early-stage kidney cancer. Urol Oncol 2017;35:153.e7-14. 9. Ristau BT, Handorf EA, Cahn DB, Kutikov A, Uzzo RG, Smaldone MC. Partial nephrectomy is not associated with an overall survival advantage over radical nephrectomy in elderly patients with stage Ib-II renal masses: an analysis of the national cancer data base. Cancer 2018;124:3839-48.

10. Bindayi A, Autorino R, Capitanio U, Pavan N, Mir MC, Antonelli A, et al. Trifecta outcomes of partial nephrectomy in patients over 75 years old: analysis of the REnal SURGery in Elderly (RESURGE) Group. Eur Urol Focus 2019;S24054569(19)30030-6.

11. Antonelli A, Veccia A, Pavan N, Mir C, Breda A, Takagi $\mathrm{T}$, et al. Outcomes of partial and radical nephrectomy in octogenarians - a multicenter international study (Resurge). Urology 2019;129:139-45.

12. Mir MC, Pavan N, Capitanio U, Antonelli A, Derweesh I, Rodriguez-Faba $\mathrm{O}$, et al. Partial versus radical nephrectomy in very elderly patients: a propensity score analysis of surgical, functional and oncologic outcomes (RESURGE project). World J Urol 2019.

13. Vartolomei MD, Matei DV, Renne G, Tringali VM, Crișan N, Musi G, et al. Long-term oncologic and functional outcomes after robot-assisted partial nephrectomy in elderly patients. Minerva Urol Nefrol 2019;71:31-7.

14. Austin PC. Optimal caliper widths for propensity-score matching when estimating differences in means and differences in proportions in observational studies. Pharm Stat 2011;10:150-61.
15. Assel M, Sjoberg D, Elders A, Wang X, Huo D, Botchway A, et al. Guidelines for reporting of statistics for clinical research in urology. J Urol 2019;201:595-604.

16. Veccia A, Autorino R. Is there a relation between preserved renal function and oncological outcomes in patients undergoing partial nephrectomy for renal cell carcinoma? Ann Transl Med 2018;6(Suppl 1):S88.

17. Buffi NM, Saita A, Lughezzani G, Porter J, Dell'Oglio $\mathrm{P}$, Amparore D, et al.; ERUS Scientific Working Group. Robot-assisted Partial Nephrectomy for Complex (PADUA Score $\geq 10$ ) Tumors: Techniques and Results from a Multicenter Experience at Four High-volume Centers. Eur Urol 2020;77:95-100.

18. Minervini A, Mari A, Borghesi M, Antonelli A, Bertolo $\mathrm{R}$, Bianchi $\mathrm{G}$, et al. The occurrence of intraoperative complications during partial nephrectomy and their impact on postoperative outcome: results from the RECORd1 project. Minerva Urol Nefrol 2019;71:47-54.

19. Kim SP, Campbell SC, Gill I, Lane BR, Van Poppel H, Smaldone MC, et al. Collaborative Review of Risk Benefit Trade-offs Between Partial and Radical Nephrectomy in the Management of Anatomically Complex Renal Masses. Eur Urol 2017;72:64-75.

20. Brown GC. Living too long: the current focus of medical research on increasing the quantity, rather than the quality, of life is damaging our health and harming the economy. EMBO Rep 2015;16:137-41.

21. Clegg A, Young J, Iliffe S, Rikkert MO, Rockwood K. Frailty in elderly people. Lancet 2013;381:752-62.

22. Venkatramani V, Koru-Sengul T, Miao F, Nahar B, Prakash NS, Swain S, et al. A comparison of overall survival and perioperative outcomes between partial and radical nephrectomy for $\mathrm{cT} 1 \mathrm{~b}$ and $\mathrm{cT} 2$ renal cell carcinoma-Analysis of a national cancer registry. Urol Oncol 2018;36:90.e9-14.

23. Denic A, Glassock RJ, Rule AD. Structural and functional changes with the aging kidney. Adv Chronic Kidney Dis 2016;23:19-28.

24. Shen J, Ruckle D, Li R, Keheila M, Alsyouf M, Schober $\mathrm{J}$, et al. How a donor nephrectomy population can help give perspective to the effects of renal parenchymal preservation during partial nephrectomy. J Endourol 2019;33:417-22.

25. Wu J, Suk-Ouichai C, Dong W, Zhang Z, Tanaka H, Wang Y, et al. Vascularized parenchymal mass preserved with partial nephrectomy: functional impact and predictive factors. Eur Urol Oncol 2019;2:97-103.

26. Nagahara A, Uemura $M$, Kawashima A, Ujike T, Fujita K, Miyagawa Y, et al. R.E.N.A.L. nephrometry score predicts postoperative recurrence of localized renal cell carcinoma treated by radical nephrectomy. Int J Clin Oncol 2016;21:367-72.

27. Bertolo RG, Zargar H, Autorino R, Fiori C, Kaouk JH, Russo $\mathrm{P}$, et al. Estimated glomerular filtration rate, renal scan and volumetric assessment of the kidney before and after partial nephrectomy: a review of the current literature. Minerva Urol Nefrol 2017;69:539-47.

28. Bertolo R, Fiori C, Piramide F, Amparore D, Barrera M, Sardo D, et al. Assessment of the relationship between renal volume and renal function after minimally-invasive partial nephrectomy: the role of computed tomography and nuclear renal scan. Minerva Urol Nefrol 2018;70:509-17.

Conflicts of interest.-The authors certify that there is no conflict of interest with any financial organization regarding the material discussed in the manuscript.

Authors' contributions.-Protocol/project development: Alessandro Veccia, Riccardo Autorino; data collection or management: Alessandro Veccia, Paolo Dell'Oglio, Giuseppe Simone, Benjamin Challacombe, Sisto Perdonà, James Porter, Chao Zhang, Umberto 


\section{COPYRIGHT $^{(\odot)} 2020$ EDIZIONI MINERVA MEDICA}

Capitanio, Chandru P. Sundaram, Giovanni Cacciamani, Uzoma Anele, Geert De Naeyer, Aaron Bradshawh, Andrea Mari, Riccardo Campi, Cristian Fiori, Ken Jacobsohn, Daniel Eun, Clayton Lau; data analysis: Alessandro Veccia, Riccardo Autorino; manuscript writing / editing: Alessandro Veccia, Paolo Dell’Oglio, Alessandro Antonelli, Andrea Minervini, Giuseppe Simone, Benjamin Challacombe, Umberto Capitanio, Giovanni Cacciamani, Uzoma Anele, Riccardo Campi, Ithaar Derweesh, Francesco Porpiglia, Riccardo Autorino; other (Supervision): Alessandro Veccia, Paolo Dell'Oglio, Alessandro Antonelli, Andrea Minervini, Giuseppe Simone, Benjamin Challacombe, Sisto Perdonà, James Porter, Chao Zhang, Umberto Capitanio, Chandru P. Sundaram, Giovanni Cacciamani, Monish Aron, Uzoma Anele, Lance J. Hampton, Claudio Simeone, Geert De Naeyer, Aaron Bradshawh, Andrea Mari, Riccardo Campi, Marco Carini, Cristian Fiori, Michele Gallucci, Ken Jacobsohn, Daniel Eun, Clayton Lau, Jihad Kaouk, Ithaar Derweesh, Francesco Porpiglia, Alexandre Mottrie, Riccardo Autorino.

Article first published online: September 13, 2019. - Manuscript accepted: September 4, 2019. - Manuscript received: July $21,2019$. 